\title{
MODELADO DEL EFECTO DE LA MOVILIDAD DEL BANCO DE FINOS DE PROPANTE SOBRE LAS TENDENCIAS DE PRODUCCIÓN EN POZOS HIDRÁULICAMENTE FRACTURADOS
}

Renso Alfredo Mayorga Ballesteros ${ }^{1 *}$, Fernando Enrique Calvete González².

* A quien debe dirigirse la correspondencia.

\section{RESUMEN}

En términos generales, el fracturamiento hidráulico es el proceso físico-mecánico de creación de fracturas en la formación productora con el propósito de generar canales de flujo altamente conductivos con el fin de acelerar la producción total del pozo. Para tal propósito, se realiza la inyección de un fluido polimérico altamente viscoso acompañado de un material particulado denominado material propante o material apuntalante, cuya función principal es la de servir como material de soporte para mantener abiertas las fracturas creadas en la roca luego de finalizar el programa de bombeo.

La aplicación cíclica de estos esfuerzos por parte de la roca que entra en contacto con el material propante de la fractura, da inicio a un proceso de desintegración gradual de las partículas mejor conocido como proceso de aplastamiento o Crushing por su nombre en inglés. Este fenómeno, da origen a la aparición progresiva de partículas de menor tamaño y morfología heterogénea(finos), las cuales tienen la capacidad de ser transportadas y depositadas espacialmente a lo largo de todo el paquete de propante, generando así zonas afectadas con una obstrucción parcial o total de los canales altamente conductivos. Este proceso de movilización y re-asentamiento de finos de propante se da en mayor o menor grado a lo largo de la vida productiva de la fractura y tiene su mayor grado de afectación, cuando se realizan procesos de estimulación química remedial logradas a través de la inyección a diferentes caudales de fluidos reactivos dentro de la fractura hidráulica.

El presente estudio presenta un nuevo enfoque de ingeniería que permite la cuantificación del impacto generado por la movilización y redistribución del banco de finos de propante sobre la alteración de la conductividad efectiva de la fractura y su producción asociada. Para tal fin se desarrolló un modelo de pozo fracturado hidráulicamente tipo sencillo (Single Well Model SWM) empleando un refinamiento local del enmallado de simulación ( Local Grid Refinement $L G R$ ) en un simulador de yacimientos comercial, sobre el cual se realizó todo el desarrollo experimental de la presente investigación, y el cual permitió recrear los diferentes patrones de distribución de forma, para obtener así las diferentes tendencias de producción luego de un evento de estimulación química remedial realizado a alto volumen y alto caudal. Los resultados obtenidos muestran porcentajes de afectación sobre la producción acumulada del orden el 9 al $30 \%$ con respecto al caso base de producción. Los resultados indican que el efecto de los diferentes patrones de distribución de forma ocasionados por la re-movilización del banco de finos de propante genera un detrimento significativo y sostenido sobre los niveles de producción, razón por la cual debe ser minimizado al máximo durante los procesos de intervención de pozo que involucren la inyección de fluidos a lo largo de la fractura deteriorada. Adicionalmente se concluyó que este tipo de intervenciones, no solo se deben realizar a tasas de inyección matricial, si no que adicionalmente sugieren un enfoque y estrategia de control similar al aplicado en formaciones con bajas tasas críticas de movilidad de finos propios de la formación.

Palabras Claves: Finos de propante, fracturamiento hidráulico, curvas de producción, esfuerzos cíclicos.

1. Grupo de investigación modelamiento de procesos de hidrocarburos - GMPH, Escuela de Ingeniería de Petróleos, Universidad Industrial de Santander. UIS, Carrera 27calle 9, Bucaramanga, Colombia. Email: renso. mayorga@hotmail.com

2. Grupo de investigación modelamiento de procesos de hidrocarburos - GMPH, Escuela de Ingeniería de Petróleos, Universidad Industrial de Santander. UIS, Carrera 27calle 9, Bucaramanga, Colombia. Email: fcalvete@uis.edu.co 


\title{
MODELING THE PROPPANT FINES MOBILITY ON PRODUCTIONS TRENDS FOR HIDRAULIC FRACTURED WELLS
}

\begin{abstract}
In general terms, hydraulic fracturing is the physical -mechanical process of creating fractures in the formation in order to provide highly conductive flow channels to accelerate the total production. To this end, the injection of a highly viscous polymer fluid accompanied by particulate material called proppant material whose main function is to keep open the fissures in the rock.

The application of efforts by the rock that comes into contact with the fracture proppant material begins a crushing process. This phenomenon gives rise to the gradual appearance of proppant fines, which have the ability to be transported and deposited spatially throughout the proppant pack, thereby generating affected areas with partial obstruction or total highly conductive channels. This process occurs throughout the productive life of the fracture and has its greatest influence during the execution of chemical stimulation treatments through the proppant pack.

This study presents a new engineering approach that enables quantification of the impact generated by the mobilization and redistribution of the proppant fines banking on altering the effective fracture conductivity and its associated production. A Single Well Model (SWM) was created using Local Grid Refinement (LGR) to represent the partial fracture conductivity reduction, this model allowed to recreate the different distribution patterns in order to obtain the production trends after a remedial chemical stimulation event. The results show percentages of detrimental production between 9 to $30 \%$ compared to the base case production. The results indicate that the effect of the various distribution patterns of form caused by the re-mobilization of proppant fines banking generates significant and sustained levels of production reduction. This negative factor should be minimized during the well intervention involving the injection of fluid along the deteriorated fracture. In addition, it was found that this type of interventions, not only must be performed at matrix injection rates, but also considering a control strategy similar to those used in wells with high production of formation's fines.
\end{abstract}

Keywords: Proppant fines, Hydraulic Fracturing, Production Trends, and Cyclic Stress.

\section{INTRODUCCION}

Desde una perspectiva integral, el éxito de un fracturamiento hidráulico es el resultado de la integración efectiva de tres etapas fundamentalmente: La primera etapa está relacionada con una adecuada selección del pozo candidato, así como una efectiva interpretación de la información técnica disponible para lograr el diseño de fractura más eficiente tanto para la condición actual del pozo como para la formación que contendrá la fractura hidráulica. La segunda etapa está relacionada con el éxito operacional alcanzado durante la ejecución del trabajo principal de fracturamiento hidráulico. Es decir, con la correcta ejecución del programa de bombeo, con el adecuado posicionamiento del material propante dentro de la geometría de fractura diseñada y con un controlado plan de finalización de bombeo que minimice las posibilidades de sobre desplazamiento del propante hacia la formación. Finalmente, la tercera etapa asociada al éxito de un fracturamiento hidráulico tiene que ver con la vida útil del nuevo sistema productivo generado. La capacidad de retornar a superficie la máxima cantidad de fluido de fractura, el control en las condiciones de arranque y estabilización del pozo, las posteriores estimulaciones químicas remediales y en general todos aquellos eventos de pozo que involucren una alteración en la dinámica de fluidos a través de la fractura hidráulica son aspectos críticos para el sostenimiento de la continuidad de la tendencia de producción del pozo intervenido.

De acuerdo con Economides \& Valkó (2003), la productividad del pozo será entre otras, función de las características de la fractura y del yacimiento. Para el caso del yacimiento, la pérdida gradual de presión en el reservorio, da origen a fenómenos de daño de formación, cambios significativos en el campo de esfuerzo en el área de drenaje, alteraciones de las permeabilidades relativas, conificación a través de las formaciones productoras y cierre de fracturas conductivas para el caso de yacimientos naturalmente fracturados entre otras. Con respecto a la fractura, Lehman, Parker, Baluch \& Haynes (1999), establecen que la conductividad, entendida como la capacidad de flujo de los fluidos del yacimiento a través del medio propante poroso, está directamente afectada por la cantidad y distribución de polímero remanente dentro de la fractura, los precipitados geomecánicos, la migración de finos de formación hacia el paquete propante, el fenómeno de embebimiento del material apuntalante en la periferia de la zona de contacto de la fractura con la formación y por la aparición de material particulado fino producto del esfuerzo cíclico de sobrecarga al que es sometido el propante a lo largo del historial de producción del pozo. 
Sumado a este escenario adverso, Guppy, Cinco-Ley \& Ramey (1982) y Giddley (1991), concluyen en su investigación que adicionalmente, el efecto de flujo no Darcy dentro de la fractura, genera una reducción en la conductividad del paquete de propante, producto de la movilización de los finos dentro del medio poroso como consecuencia del régimen turbulento inducido en eventos de alteración súbita del diferencial de presión de producción (Drawdown).

Por su parte, Cinco-Ley \& Samaniego (1977) y CincoLey, Samaniego \& Domínguez (1978), introdujeron dos valiosos conceptos relacionados con el impacto negativo sobre la producción del pozo ocasionado por del deterioro de la conductividad del paquete de propante en la periferia del contorno externo de la fractura (Fracture Face Skin- $S_{f f}$ ) y en el área de contacto entre la fractura y los perforados de producción del pozo (Choked Fracture Skin- $S_{c k}$ ). La identificación de esta condición de deterioro unida al concepto de movilidad y acumulación de finos de propante desarrollada por Azari (1991), dan fundamento al desarrollo de la presente investigación en la cual se introduce el concepto de patrón de distribución de forma y sus repercusiones sobre las tendencias de producción de pozos hidráulicamente fracturados los cuales han sido sometidos a un proceso de estimulación química remedial realizado a altos regímenes de flujo.

\section{METEORIZACIÓN DEL PROPANTE Y GENERACIÓN DE FINOS}

El término en inglés Crushing, ampliamente utilizado en la industria de los hidrocarburos, puede traducirse al español, como aplastamiento, o puede también abordarse desde el punto de vista geológico para hacer una referencia análoga con el fenómeno de meteorización física descrito por Monkhouse (1978) quien señala que el proceso de meteorización física produce desintegración o ruptura de la roca (en nuestro caso el propante), sin alterar su composición química o mineralógica.

De acuerdo con el ensayo de evaluación de propantes descrito en la norma API RP 19C - Sección 11 (2008), cuando el material evaluado alcanza el punto de falla, las esferas de propante colapsan para formar partículas amorfas de menor tamaño. Estas partículas alcanzan en promedio un tamaño inferior al de la malla 100 y son considerados finos de propante. Figura 1. La habilidad de los finos para migrar a través del paquete de propante depende tanto del tamaño de partícula, como de la estructura de este. Dicho proceso de migración genera puenteo granular y obstrucción de las gargantas porales, lo que deteriora la capacidad de flujo a través de los canales preferenciales.

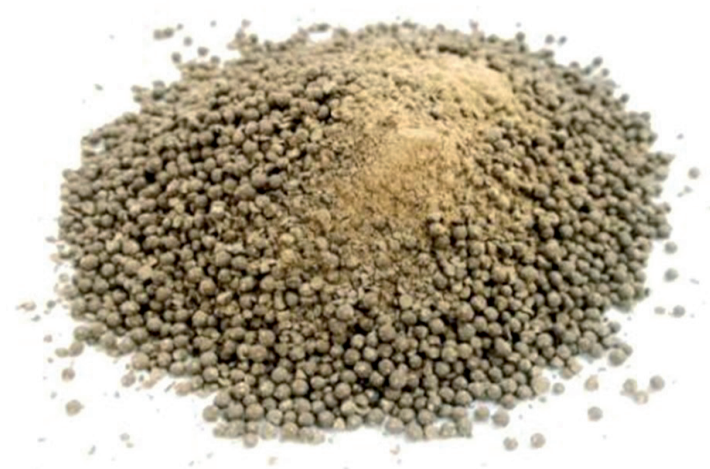

Figura 1. Finos de Propante-Norma API-RP19C.

Fuente: El autor

Esta capacidad de flujo a través de la fractura, se representa a través del concepto de conductividad adimensional de la fractura (1) planteado por CincoLey \& Samaniego (1977) y Cinco-Ley et al. (1978), el cual, hasta la fecha es uno de los criterios de diseño y evaluación de mayor impacto sobre el índice de productividad de los pozos fracturados.

$$
C_{f D}=\frac{w_{a v e} k_{f}}{x_{f}{ }^{*} k}
$$

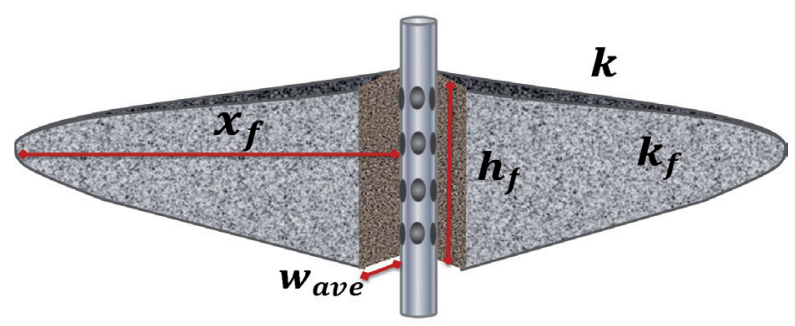

Figura 2. Parámetros de Geometría de Fractura. Fuente: El autor

Donde:

$C_{f D \text { : Es la conductividad de fractura adimensional }}$ $W_{\text {ave: }}$ Es el ancho promedio de la fractura empaquetada en $\mathrm{ft}$.

$k_{f}$ : Es la permeabilidad de la fractura en $\mathrm{mD}$.

$h_{f}$ : Es la altura de la fractura en $\mathrm{ft}$.

$x_{f}$ : Es la longitud media de la fractura en $\mathrm{ft}$. Y

$k$ : Es la permeabilidad de la matriz en $\mathrm{mD}$. 


\section{PATRÓN DE DISTRIBUCIÓN DE FORMA}

El presente estudio introduce el concepto de patrón de distribución de forma, el cual representa la distribución probabilística del banco de propantes de finos dentro del espacio poral del paquete de propante de la fractura hidráulica.

La generación de esfuerzos cíclicos de la formación sobre la fractura, incrementan gradualmente la aparición del banco de finos de propante como lo demuestran los resultados consignados en la Tabla 1, correspondientes al ensayo de meteorización realizado en un laboratorio de fluidos y materiales de fractura, empleando bauxita referencia 12/18 como agente propante y aplicando 4 ciclos de 7,500psi de carga uniaxial en la celda estándar para confinamiento y generación de esfuerzos.

Tabla1.Resultados del ensayo cíclico de aplastamiento.

\begin{tabular}{|ccccc|}
\hline \multicolumn{5}{|c|}{ EVALUACIÓN DE CARGA CÍCLICA } \\
$\begin{array}{c}\text { Contenido } \\
\text { Inicial de } \\
\text { finos }(\mathrm{g})\end{array}$ & $\begin{array}{c}\text { Primer } \\
\text { Ciclo }(\mathrm{g})\end{array}$ & $\begin{array}{c}\text { Segundo } \\
\text { Ciclo }(\mathrm{g})\end{array}$ & $\begin{array}{c}\text { Tercer } \\
\text { Ciclo }(\mathrm{g})\end{array}$ & $\begin{array}{c}\text { Cuarto Ciclo } \\
(\mathrm{g})\end{array}$ \\
\hline 0 & 2.53 & 7.72 & 11.03 & 14.26 \\
\hline $\begin{array}{c}\text { Porcentaje de } \\
\text { Finos }(\%)\end{array}$ & 6.02 & 18.38 & 26.91 & 33.95 \\
\hline
\end{tabular}

Como lo ilustra la Figura 3, hay una fuerte tendencia incremental en la generación de material particulado con cada ciclo de esfuerzo que se induce sobre el material apuntalante. Adicionalmente, durante la realización del presente ensayo, se pudo observar que luego de cada ciclo, el material particulado disminuía gradualmente su tamaño, hasta alcanzar una morfología semi-esférica de 300 micrones de diámetro aproximadamente. Lo cual desde luego favorece su capacidad de movilidad dentro del paquete de propante.

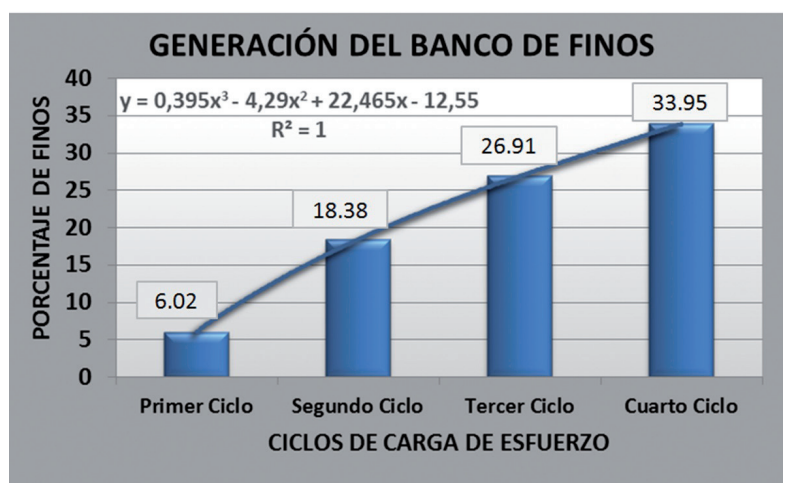

Figura 3. Tendencia de formación de Finos Fuente: El autor
La movilidad del banco de finos es generada por el transporte inducido bien sea por los fluidos de producción o por los sistemas de remediación de daño de formación los cuales son inyectados en la fractura, en dirección opuesta al flujo de producción. Esta condición de flujo bidireccional, sumada al efecto turbulento producto del flujo No Darcy ocasiona la migración y redepositación por efecto gravitacional a lo largo y ancho de la fractura hidráulica, generando, por consiguiente, zonas preferenciales de depositación que actuarán como barreras adicionales para la movilidad de las partículas hacia las proximidades de la cara del pozo.

El fenómeno anteriormente descrito, genera patrones de forma distribuidos dentro del paquete de propante que finalmente se traducirán en una reducción significativa en los valores de la conductividad adimensional de la fractura.

A continuación, se ilustran dos patrones de distribución de forma logrados a nivel de laboratorio, los cuales representan dos escenarios probables luego de un evento de estimulación química remedial.

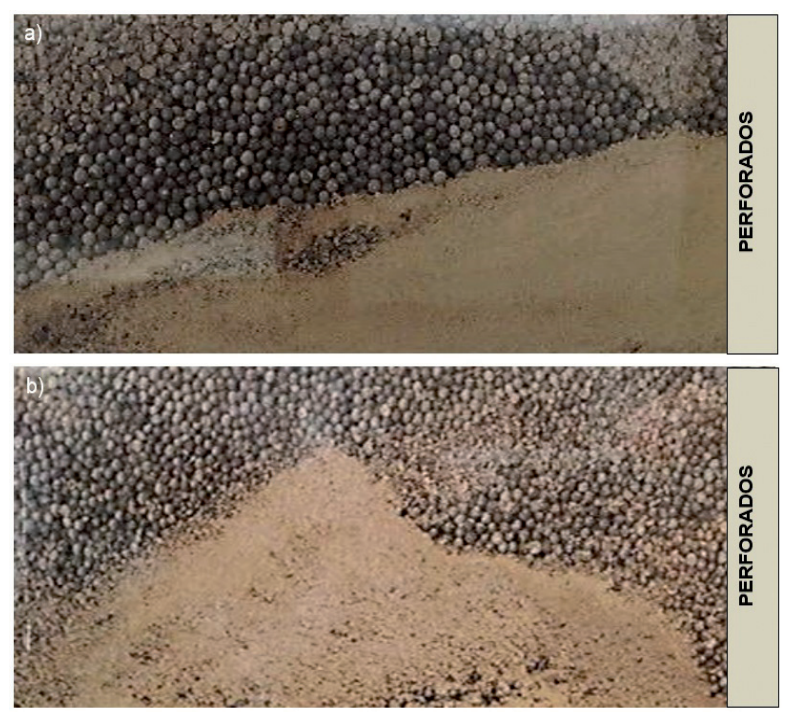

Figura 4. a) y b) Primer y Segundo Patrón de

Distribución de Finos de propante generados a nivel de laboratorio.

Fuente: El autor

\section{MODELO DE SIMULACIÓN}

Para realizar la cuantificación del efecto de los patrones de distribución de finos sobre la tendencia de producción se construyó un modelo de producción tipo pozo sencillo (Single Well Model), empleando un simulador de yacimientos basado en el desarrollo de diferencias finitas. 
El pozo representado mediante el modelo de simulación corresponde a un pozo productor de gas condensado ubicado en los llanos orientales colombianos. Este pozo fue perforado a 15,298 pies de profundidad en un yacimiento composicional, naturalmente fracturado, el cual produce aceite, gas y agua en flujo natural a través de sus 4 formaciones productoras.

La construcción de este modelo se hizo con un enmallado tipo cartesiano, el cual permite modelar el flujo lineal de los fluidos en patrones con áreas grandes con celdas refinadas en las proximidades de la cara del pozo. Las dimensiones del enmallado son: $15 \mathrm{i}, 15 \mathrm{j}$, $21 \mathrm{k}$, para un total de 5,490 bloques, con dimensiones de 200 pies en las direcciones i-j (0.92 Acres ó 3,716 $\mathrm{m}^{2}$ ). El área total del modelo es de 83.6 acres, donde las 21 capas en dirección $\mathrm{k}$ representan las diferentes formaciones constitutivas del yacimiento como se referencian a continuación: Primera formación (capas 1 a la 10), primer sello (capa 11), segunda formación (capas 12 a la 15), segundo sello (capa16) y tercera formación (capas 17 a la 21). La fractura hidráulica está contenida en la primera formación, la cual posee las propiedades petrofísicas más favorables del yacimiento. La Figura 5 contiene el modelo base de simulación descrito anteriormente.

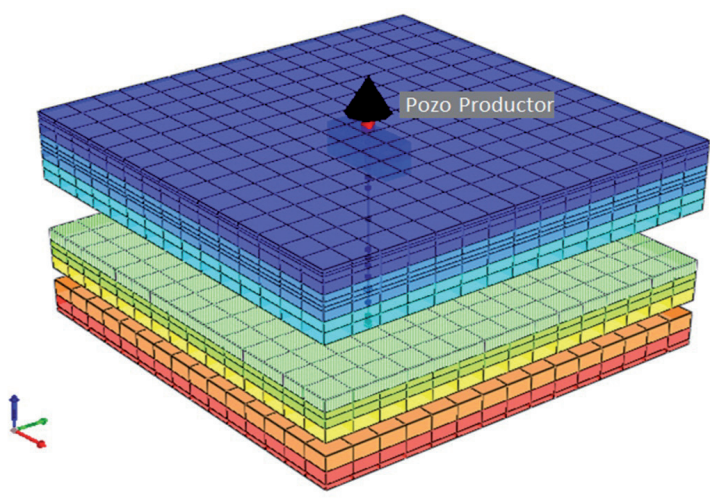

Figura 5. Modelo de simulación tipo pozo sencillo fracturado hidráulicamente.

Fuente: Computer Modeling Group, CMG.

Para lograr una mayor representatividad de los resultados obtenidos, se desarrolló una completa arquitectura del modelo de simulación como se muestra a continuación:

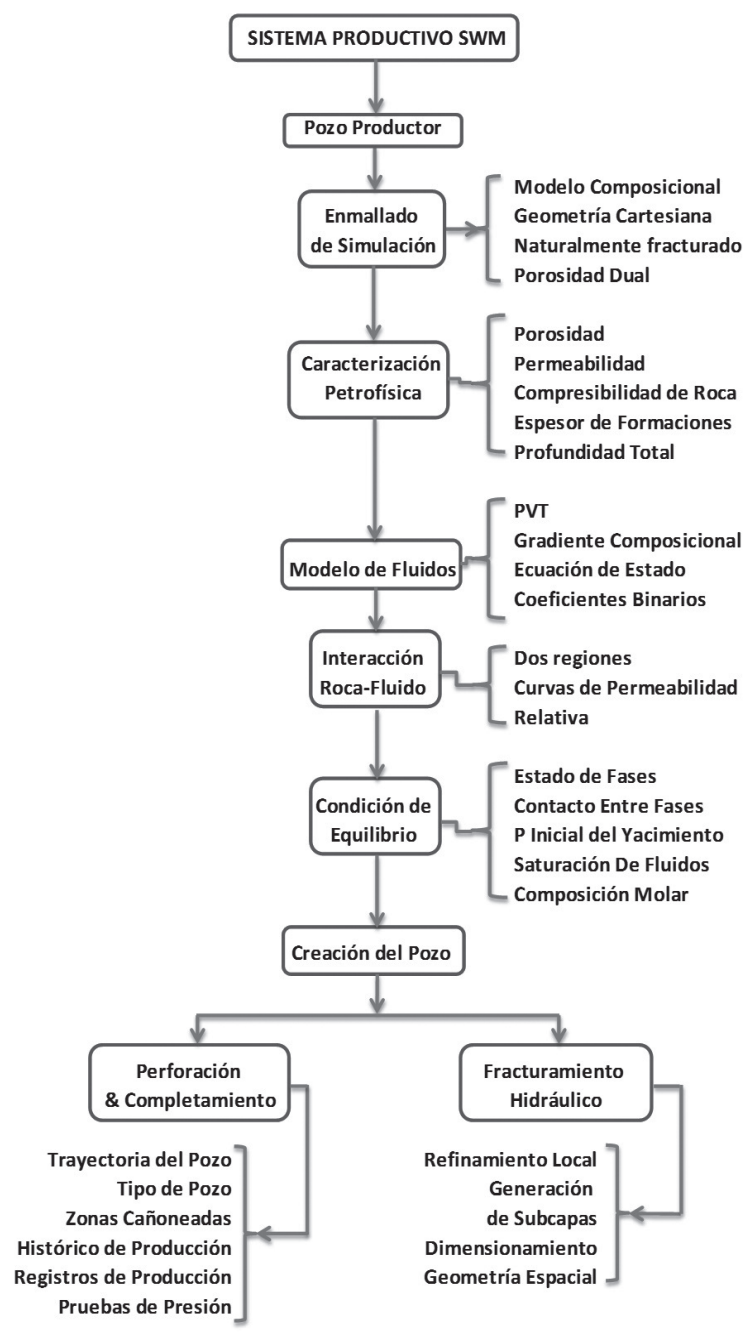

Figura 6. Arquitectura del Modelo $S W M$. Fuente: El Autor.

Para generar la fractura hidráulica dentro del modelo de simulación, se subdividieron las capas principales de la formación superior con el propósito de lograr un ajuste perfecto de las dimensiones de la fractura. De igual modo se realizó un refinamiento local del enmallado en las proximidades de los perforados con el objetivo de generar los diferentes patrones de distribución de forma dentro de la geometría de la fractura. A continuación, se presentan los parámetros de diseño empleados en la generación de la fractura. 
Tabla2. Diseño de Ingeniería para el modelado del fracturamiento hidráulico.

\begin{tabular}{|c|c|c|}
\hline \multicolumn{3}{|c|}{ PARÁMETROS DE DISEÑO DE FRACTURA } \\
\hline Intervalos Perforados & $\begin{array}{l}13,705 \\
13,765\end{array}$ & pies \\
\hline Temperatura de Yacimiento & 235 & ${ }^{\circ}$ Fahrenheit \\
\hline $\begin{array}{l}\text { Permeabilidad promedio } \\
\text { Matriz }\end{array}$ & 3.282 & $\mathrm{mD}$ \\
\hline $\begin{array}{l}\text { Concentración del Fluido de } \\
\text { Fractura }\end{array}$ & 4.5 & libras/galón \\
\hline $\begin{array}{l}\text { Concentración Max. de } \\
\text { propante }\end{array}$ & 10 & libras/galón \\
\hline Longitud Media de Fractura & 118 & pies \\
\hline Altura Superior de Fractura & 129 & pies \\
\hline Altura Inferior de Fractura & 140 & pies \\
\hline $\begin{array}{l}\text { Longitud de Fractura } \\
\text { Empaquetada }\end{array}$ & 118 & pies \\
\hline $\begin{array}{l}\text { Ancho de Fractura en } \\
\text { Perforados }\end{array}$ & 0.145 & pies \\
\hline $\begin{array}{l}\text { Concentración Promedio de } \\
\text { Propante }\end{array}$ & 6.29 & libras/pie2 \\
\hline Conductividad de Fractura & 35.2 & Adimensional \\
\hline Módulo de Young & $8^{\prime} 300,000$ & psi \\
\hline Relación de Poisson & 0.31 & Adimensional \\
\hline Compresibilidad Total & 4.15E-07 & $1 / \mathrm{psi}$ \\
\hline
\end{tabular}

A continuación, se procede a estimar la permeabilidad de la fractura hidráulica a partir de la ecuación de CincoLey \& Samaniego (1977) y Cinco-Ley et al. (1978):

$$
\begin{gathered}
k_{f}=\frac{C_{f D} * x_{f} * k}{W_{\text {ave }}} \\
k_{f}=\frac{35.2 * 118 * 3.282}{0.145}=94,014.6 \mathrm{mD}
\end{gathered}
$$

Al realizar el remplazo de los valores de la Tabla 2 en la Ecuación (2), se obtiene el valor de la permeabilidad de fractura como se indica en la Ecuación (3). A partir de este resultado, se procede a crear la fractura hidráulica en el enmallado de simulación con la geometría y propiedades especificadas en la Tabla 2. A continuación se presenta una imagen tridimensional del modelo, en donde se puede apreciar la representación de la fractura hidráulica en la formación superior del yacimiento.

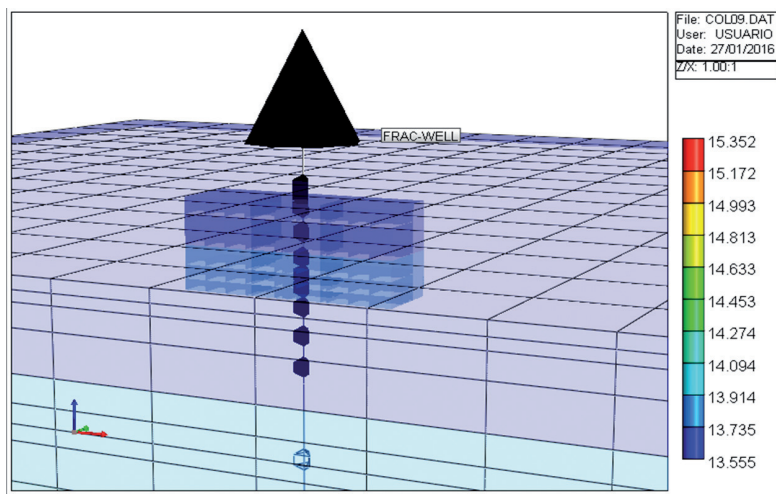

Figura 7. Fractura Hidráulica Generada en la formación superior del Modelo de Simulación.

Fuente: Computer Modeling Group, $C M G$.

\section{PATRÓN DE DISTRIBUCIÓN DE FORMA}

La siguiente etapa del proceso consiste en la generación de los patrones de distribución de forma, los cuales representarán la movilidad del banco de finos de propante dentro de la fractura. Inicialmente se considera que este banco de finos (representado para cada caso de la Figura 8 con celdas amarrillas), se encuentra uniformemente espaciado dentro del paquete de propante. La Figura 8.A, representa el caso base de un proceso de meteorización del propante in-situ bajo una condición de esfuerzo ejercido por la formación (para este caso será el esfuerzo principal sobre la fractura, el cual corresponde al esfuerzo horizontal mínimo) y a una tasa de producción en régimen de flujo laminar (Reynolds <2,000) sin generación de arrastre de partículas. El segundo patrón de distribución del banco de finos de propante corresponde a la geometría representada en la sección b de la Figura 8. Para esto, se alteran las celdas del modelo contenidas en el espacio medio inferior de la fractura representada en la Figura 7. Por último, se genera el patrón de distribución tipo gaussiano (Figura 8c), el cual representa el estado final del banco de finos de propante luego de realizada una estimulación matricial a un régimen de flujo turbulento (25-35 barriles por minuto) y, posterior a la etapa de retorno de fluido de estimulación gastado a superficie (Back Flow Stage). 
Es importante resaltar que, durante el periodo de cierre del pozo y posterior inicio del bombeo de inyección de tratamientos químicos, se reduce el esfuerzo de cierre sobre la fractura dando lugar al proceso de reacomodamiento de partículas por efectos gravitacionales, lo que resulta aún más perjudicial para la conductividad adimensional de fractura en la región media inferior del paquete de propante.

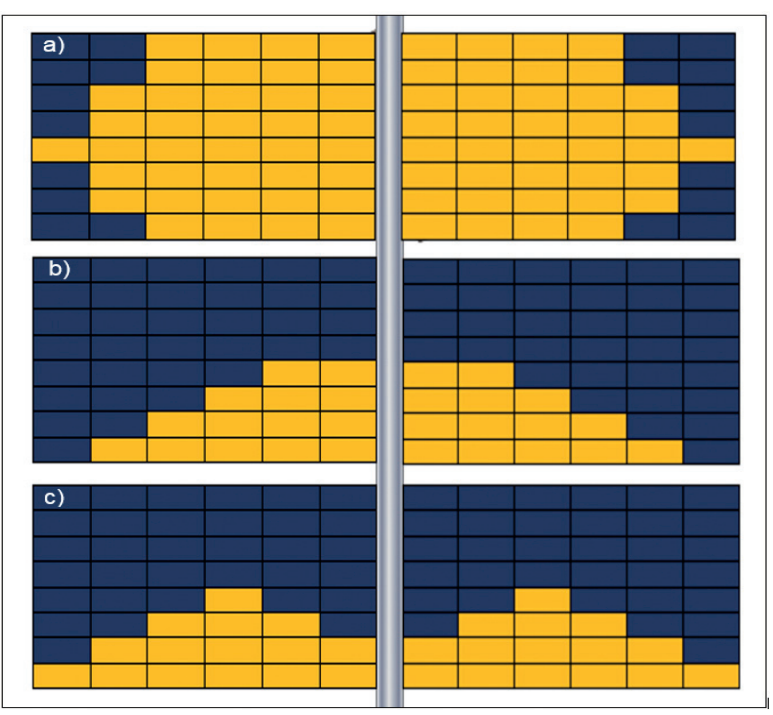

Figura 8. Patrones de Distribución de Forma Contenidos Dentro de la Fractura Hidráulica.

Fuente: El Autor.

\section{ANÁLISIS DE RESULTADOS}

Se llevaron a cabo de forma individual cada una de las corridas de simulación empleando un simulador de yacimientos comercial, obteniendo como resultado, los siguientes perfiles de producción para cada uno de los patrones de distribución de forma indicados en el apartado anterior:

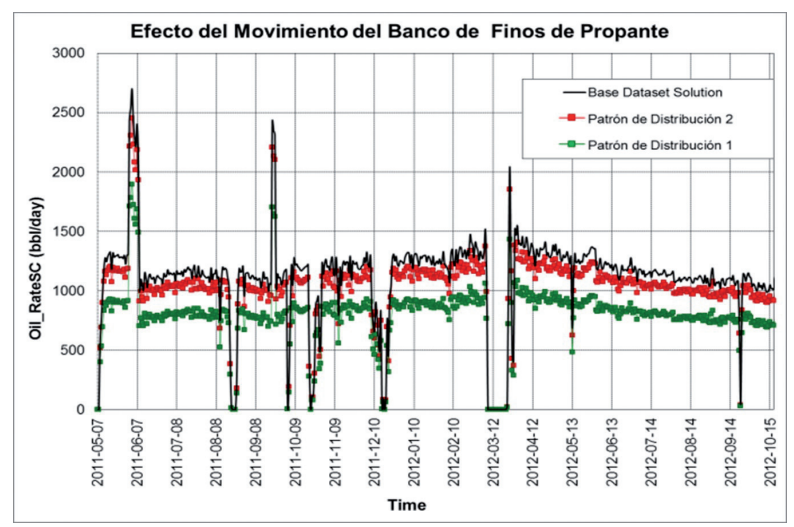

Figura 9. Curvas de Producción para Cada uno de Los Patrones de Distribución de Finos de Propante Fuente: Computer Modeling Group, CMG.
El escenario recreado corresponde al evento de cierre de pozo realizado el 5 de Mayo de 2011, para la ejecución de un trabajo de estimulación química remedial realizado a alto caudal (Movilización del banco de finos en dirección del yacimiento) cuyo objetivo era remover el daño de formación en la región que contiene la fractura hidráulica. Luego de realizada esta intervención, se reanuda la producción del pozo para retornar a superficie los remanentes de tratamiento químico gastado (Segundo evento de movilización del banco de finos en dirección de la producción). El periodo de evaluación se extiende por 17 meses hasta el 16 de octubre de 2012 con el propósito de conocer la respuesta de producción en el corto y mediano plazo. Este periodo contiene 6 eventos de cierre de pozo (Producción cero) correspondientes a eventos operacionales y de logística.

En la Figura 9 se puede observar la curva de producción representada por una línea de color negro, la cual corresponde a la producción del caso base, es decir, a la condición natural de la fractura hidráulica sin la presencia del banco de finos de propante. La línea en verde representa el patrón de forma de la Figura $8 \mathrm{~b}$ (Patrón de Distribución 1), y finalmente en rojo, el patrón de distribución tipo Gaussiano (Patrón de Distribución 2).

En primer lugar, se encontró que la forma geométrica del patrón de distribución de finos de propante dentro de la fractura hidráulica juega un papel muy relevante en los niveles de producción del pozo, puesto que para el caso del patrón de Distribución 1, se alcanzó un porcentaje de pérdida de producción del $29.8 \%$ con respecto a la producción del caso base, mientras que para el caso 2, el patrón de distribución de finos solamente afectó la producción en un $9.1 \%$.

De acuerdo con los resultados registrados en la Figura 10, los niveles de pérdida de producción con respecto al caso base de simulación son del orden de 175,809 bbl para el caso del patrón de forma progresivo (Figura $8 \mathrm{~b})$ y de menos $53,719 \mathrm{bbl}$ para el factor de forma tipo gaussiana representado en la Figura $8 \mathrm{c}$ para un periodo de producción inferior a 2 años. Está marcada diferencia se genera a partir de la condición de obstrucción de los perforados ubicados en la tubería de producción, puesto que si bien, ambos patrones afectan considerablemente los canales de flujo preferencial dentro del paquete de propante, para el caso del patrón de forma progresivo, la obstrucción de los orificios de drenaje de la fractura hacia la tubería de producción (perforados) poseen una mayor tortuosidad y bloqueo debido al efecto puenteante de las partículas de finos de propante depositadas en la zona media inferior del área efectiva de producción de la fractura hidráulica. 


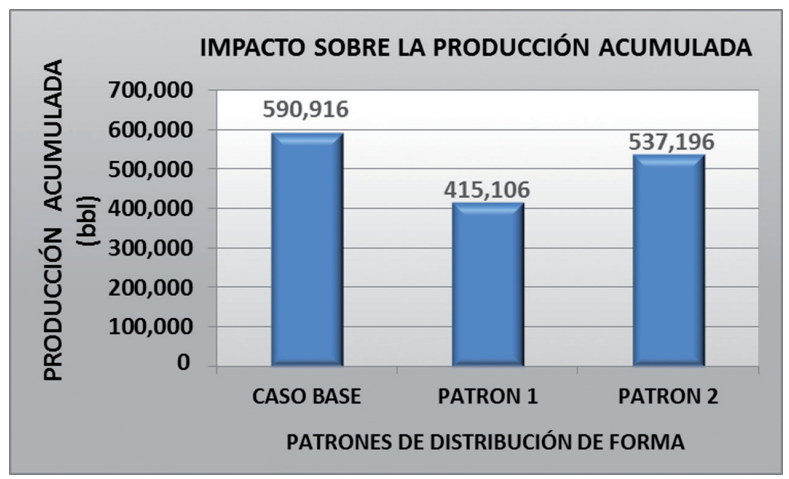

Figura 10. Resultados de Producción Acumulada para Cada uno de los Escenarios Recreados en el Simulador.

Fuente: El Autor.

Adicionalmente, la depositación preferencial de estos bancos de finos de propante, dan origen a la aparición de regiones altamente favorables para el inicio del proceso geoquímico de la diagénesis, el cual genera un daño irreversible mayor en el paquete de propante, ya que obstruye de forma permanente los canales de flujo como consecuencia de la solidificación de material cementante creado a partir de las partículas de finos de propantes bajo condiciones de presión y temperatura de fondo de pozo.

\section{CONCLUSIONES}

Los resultados obtenidos indican que efectivamente la estrategia de afectación parcial por sectores dentro del enmallado refinado del modelo de simulación sí representa alteraciones significativas en los niveles de respuesta de la función objetivo. Lo que permite inferir que el fenómeno de movilización y redistribución del banco de finos de propante sí puede ser considerado como uno de los factores primarios que originan la pérdida de productividad en pozos hidráulicamente fracturados.

El patrón de distribución del banco de finos de propante puede plantearse como una valiosa herramienta de ajuste histórico en modelos de simulación, que requieran un mayor grado de precisión en proyectos de evaluación de viabilidad de intervención de pozos candidatos a estimulación química, e incluso a procesos de restauración de permeabilidad efectiva empleando el método de re-fracturamiento hidráulico.

Los patrones geométricos de distribución de forma producto de procesos de meteorización y migración de partículas de propante, también deben ser considerados en la evaluación técnico económica de campañas de fracturamiento hidráulico y estimulación química remedial.

\section{REFERENCIAS}

1. AMERICAN PETROLEUM INSTITUTE Recommended Practice 19C ISO 13503-2, "Measurement of Properties of Proppants Used in Hydraulic Fracturing and Gravel Packing Operations". Section 11. Proppant Crush-Resistance Test. Washington D.C. U.S. May 2008. P. 23-27.

2. AZARI M., "Performance Prediction for FiniteConductivity Vertical Fractures" Society of Petroleum Engineers, SPE-22659. SPE Annual Technical Conference and Exhibition. Dallas, Texas, U.S. October 6-9, 1991. P. 2, 3, 4, 6.

3. CINCO-LEY H., SAMANIEGO V.F., "Effect of Wellbore Storage and Damage on the Transient Behavior of Vertically Fractured Wells". Society of Petroleum Engineers, SPE- 6752. Mexican Petroleum Institute. Denver, Colorado, U.S. 9-12 October 1977. P.2, 3, 6 .

4. CINCO-LEY H., SAMANIEGO V.F., DOMINGUEZ N., "Transient Pressure Behavior for a Well with Finite Conductivity Vertical Fracture". Society of Petroleum Engineers, SPE-6014-PA. Mexican Petroleum Institute. Ciudad de México. México. August, 1978. P.3, 4.

5. ECONOMIDES M., VALKÓ P. "Optimization of the Productivity Index and Fracture Geometry of a Stimulated Well with Fracture Face and Choke Skins". Society of Petroleum Engineers, SPE-81908-PA. University of Houston. Houston, Texas, U.S. February 2003. P.1.

6. GIDLEY J.L., "AMethod for Correcting Dimensionless Fracture Conductivity for Non-Darcy Flow Effects". Society of Petroleum Engineers, SPE-20710-PA. John L. Gidley and Associates. Inc. Houston, Texas, U.S. November 1991. P.1, $2,5$.

7. GUPPY K.H., CINCO-LEY H., RAMEY H.J., "Non - Darcy Flow in Wells with Finite-Conductive Vertical Fractures". Society of Petroleum Engineers, SPE8281-PA. Standford University, Society of Petroleum Engineers Journal. Standford, California, U.S. October 1982. P.3, 5.

8. LEHMAN L., PARKER M., BALUCH M., HAYNES R., "Proppant Conductivity - What Counts and Why". Society of Petroleum Engineers, SPE-52219. Halliburton Energy Services, Inc. Mid-Continent Operations Symposium. Oklahoma City, Oklahoma, U.S. March 1999. P.2, 3, 4.

9. MONKHOUSE E. J. Diccionario de términos geográficos. Barcelona: Oikos Tau-Editores. (1978). p. 300 . 


\section{TABLA DE CONVERSIÓN DE UNIDADES}

\begin{tabular}{cccc} 
Cantidad & Nombre & Símbolo & Valor en S.I.U \\
\hline \multirow{2}{*}{ Longitud } & Pie & $\mathrm{ft}$ & $0.3048 \mathrm{~m}$ \\
& Pulgada & in & $0.0254 \mathrm{~m}$ \\
\hline \multirow{2}{*}{ Presión } & $\begin{array}{c}\text { Libra por Pulgada } \\
\text { Cuadrada }\end{array}$ & $\mathrm{psi}$ & $6,894.76 \mathrm{~kg} / \mathrm{m} \cdot \mathrm{s} 2$ \\
& Acre & $\mathrm{ac}$ & $4,046.86 \mathrm{~m} 2$ \\
Área & Pie Cuadrado & $\mathrm{ft} 2$ & $0.09290 \mathrm{~m} 2$ \\
\hline \multirow{2}{*}{ Volumen } & Barril & bbl & $0.11924 \mathrm{~m} 3$ \\
& Galón & gal & $3.78 \mathrm{E}-03 \mathrm{~m} 3$ \\
\hline Masa & Libra & lb & $753.59 \mathrm{~g}$ \\
\hline Temperatura & ${ }^{\circ}$ Fahrenheit & ${ }^{\circ} \mathrm{F}$ & $-17.222{ }^{\circ} \mathrm{C}$ \\
\hline
\end{tabular}

Recepción: 31 de agosto de 2016

Aceptación: 5 de octubre de 2016 